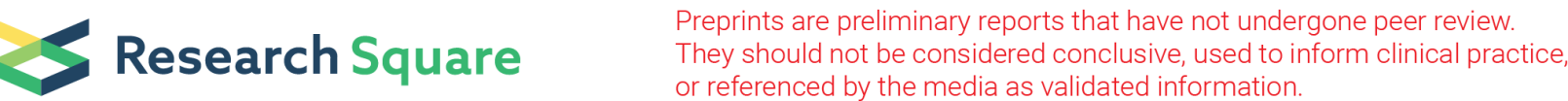

\section{Improved Survival After Multidisciplinary Team Decision-Making for Patients with Advanced Gastrointestinal Cancer: A Multicenter, Prospective, Noninterventional, Controlled Study}

Qi Zhang

Peking University School of Oncology, Beijing Cancer Hospital \& Institute

Ye Zhou

Fudan University Shanghai Cancer Center

Lijie Song

The First Affiliated Hospital of Zhengzhou University

Weijia Fang

Zhejiang University

Meng Qiu

Sichuan University

Yanhong Gu

The First Affiliated Hospital of Nanjing Medical University

Yang Yang

Cancer Institute of Nanjing University

Jingdong Zhang

Liaoning Cancer Hospital \& Institute, Cancer Hospital of China Medical University

Jing Liu

The First Hospital of China Medical University

Ming Lu

Peking University School of Oncology, Beijing Cancer Hospital \& Institute

Tianxiao Gong

Second Affiliated Hospital of Zhengzhou University

Xin Wang

Sichuan University

Yan Li

Academician Workstation, The Third People's hospital of Zhengzhou

Jun Yang

Port Hospital of Hebei Port Group Co.Ltd.

\section{Yingjiang Ye}

Peking University People's Hospital 


\section{Lin Shen}

Peking University School of Oncology, Beijing Cancer Hospital \& Institute

Jian Li ( $\sim$ oncogene@163.com )

Peking University School of Oncology, Beijing Cancer Hospital \& Institute

\section{Research Article}

Keywords: multidisciplinary team, advanced gastrointestinal cancer, survival benefit

Posted Date: December 16th, 2021

DOI: https://doi.org/10.21203/rs.3.rs-1102160/v1

License: (c) (1) This work is licensed under a Creative Commons Attribution 4.0 International License.

Read Full License 


\section{Abstract}

\section{Background}

Formal multidisciplinary team (MDT) discussions in clinical practice require time and space with unclear survival benefits for advanced gastrointestinal patients. This study aimed to investigate the long-term survival of patients with advanced gastrointestinal cancer after multidisciplinary team (MDT) decisionmaking.

\section{Materials and Methods}

From June 2017 to June 2019, continuous MDT discussions on advanced gastrointestinal cancer were conducted in ten medical centers in China. MDT decisions and actual treatment received by patients were prospectively recorded. The primary endpoint was the difference in overall survival (OS) between patients in MDT decision implementation and nonimplementation groups. The secondary endpoints included the implementation rate of MDT decisions and subgroup survival analysis.

\section{Results}

A total of 461 MDT decisions of 455 patients were included in this study. The implementation rate of MDT decisions was $85 \cdot 7 \%$. Sex and previous treatment had an impact on MDT decision-making. The OS was 24.0 months and 17.0 months, respectively, in MDT decision implementation and nonimplementation groups. The implementation of MDT decisions significantly reduced the risk of death in the univariate analysis. The subgroup analysis showed a significant difference in survival analysis of patients with colorectal cancer, but no significant difference was found in patients with gastric cancer. The rate of secondary MDT discussion was very low.

\section{Conclusion}

MDT discussion can prolong the OS of patients with advanced gastrointestinal cancer, especially colorectal cancer. Scheduling of the next MDT discussion in time is necessary when the disease condition changes.

\section{Introduction}

Gastrointestinal cancer is a high-incidence tumor in the world[1]. It is difficult for clinical guidelines to provide the most appropriate individualized treatment recommendations for each patient, especially for advanced patients, due to the diversity of clinicopathological features and heterogeneity of molecular characteristics of gastrointestinal tumours. Therefore, multidisciplinary cooperation is needed to provide the most reasonable recommendations for patients.

Multidisciplinary team (MDT) originated from the UK in 1995, with a report recommending the coordination within MDT regarding the treatment of colorectal cancer[2]. MDT is usually composed of 
specialists from two or more related disciplines, which discuss and formulate patient-specific treatment recommendations[3]. After decades of development, several countries have formed a relatively perfect MDT[4-6] and even recommended all patients with newly diagnosed cancer to undergo MDT discussion. However, only few studies have been conducted to track the follow-up implementation of MDT decisions and final overall survival ${ }^{7-9}$. In clinical practice, only a few patients receive formal MDT decisions due to the limitation of time and space. However, many patients who have not received formal MDT decisions still get multidisciplinary treatment under the doctor's supervision. Therefore, whether a formal MDT discussion can bring more benefits to patients in the current situation needs further exploration. At the same time, no prospective controlled study with a large sample size evaluated what the best MDT discussion mode was and how much survival benefits the MDT mode could bring to patients with advanced cancer.

Since MDT discussion has been gradually applied in clinical practice, it is very difficult to carry out an analysis with randomized controlled trial. Therefore, a prospective MDT study was conducted to evaluate the impact of MDT decision-making on the survival of patients with advanced gastrointestinal cancer by grouping them according to their implementation of MDT decisions.

\section{Patients And Methods}

\section{Study design and patients}

This was a prospective, noninterventional, controlled study on MDT of metastatic gastrointestinal cancer (Clinicaltrial.gov registration number: NCT03400657), which was conducted in ten medical centers with years of MDT experience in China from June 2017 to May 2019. Continuous MDT discussions were conducted in various centers. The process involving the use of patient data was in line with international ethical principles. The study was approved by the Beijing Cancer Hospital ethics committee.

Eligible patients had to have pathologically confirmed metastatic gastrointestinal malignancies. Patients had to be adults ( $\geq 18$ years of age) with an Eastern Cooperative Oncology Group performance status of 0-2, a life expectancy of at least 12 weeks, and adequate bone marrow, liver, and renal function before MDT discussion. And they have to participated in formal MDT discussion with more than two disciplines participation in the discussion. Patients with early or locally advanced tumour, primary malignant tumor other than gastrointestinal cancer, or uncontrolled medical disorders were excluded. Full inclusion and exclusion criteria are shown in the appendix. All patients provide written informed consent before enrolment.

The patients were divided into two groups according to the consistency between the MDT decision and the patient's subsequent actual treatment choice, including MDT decision implementation group (the patient's subsequent actual treatment choice was completely consistent with the MDT decision) and nonimplementation group (the patient's subsequent actual treatment choice was completely inconsistent or incompletely consistent with the MDT decision). Each decision of one patient was the unit of analysis. 


\section{Mdt Procedure}

MDT discussion application was submitted by the attending doctor, and the discussion was carried out under the MDT conventional mode of each center. The basic members of MDT discussion on gastrointestinal cancer included oncologists, gastrointestinal surgeons, hepatobiliary surgeons, radiotherapy doctors, pathologists, radiologists, and invited doctors from other departments according to the needs of patients' conditions. Each center discussed the diagnosis and treatment plan of patients in fixed place and time. The data on the disease of patients, MDT application disciplines, participating disciplines, MDT discussion time, MDT final decision, and final decision discipline were collected prospectively. After the MDT discussion, the researchers followed up on the treatment status of each patient, reasons for refusing MDT decisions, and survival status.

\section{Statistical analysis}

The primary endpoint was overall survival (OS), defined as the time from MDT discussion to death from any cause. The final known date of survival was used as the censoring date for patients who were not reported to have died at the planned analysis cutoff. The secondary endpoints included the implementation rate of MDT decisions, reasons for not implementing MDT decisions, and subgroup analysis of different tumor types. At the same time, a reasonable MDT discussion mode was also explored in this study.

Taking into consideration the current medical situation in China, it was estimated that the proportion of patients who implemented MDT decisions was about $70 \%$. Assuming the hazard ratio (HR) of 0.70 for the MDT decision implementation over nonimplementation group, it would be necessary to include approximately 421 patients (implementation group: 295 patients; nonimplementation group:126 patients) to provide an $80 \%$ statistical power with a two-sided $P$ value of 0.05 indicating statistical significance, considering about $15 \%$ loss to follow-up.

Statistical analyses were carried out using SPSS version 26.0 (SPSS Inc., IL, USA). Categorical outcomes were reported as the number and percentage and analyzed with the chi-square test, while continuous outcomes were analyzed with the parametric or nonparametric test. OS was estimated using the KaplanMeier method, described using median and $95 \%$ confidence interval $(\mathrm{Cl})$, and compared with the log-rank test. The follow-up was calculated by reverse Kaplan-Meier estimation. Cox proportional hazards regression models were used to estimate $\mathrm{HR}$ and $95 \% \mathrm{Cl}$ for variables associated with OS. The univariate Cox analysis was used to assess the association of baseline parameters with OS, and then variables with $P$ values $<0.05$ were entered into the multivariate Cox regression model, after considering collinearity among variables using a correlation matrix. The assumption of proportionality was checked using Schoenfeld partial residual plots. The cases in the majority category of a given covariate, with missing information in any of the categorical covariates [way to pay medical care (3.0\%)], were included to limit the degree of freedom of the models. And $P$ values of less than 0.05 will be taken to indicate statistically significant differences. 


\section{Results}

\section{Patient characteristics}

A total of 702 MDT discussions among 675 patients were conducted from June 2017 to May 2019; 27 patients went through second MDT discussions (Figure. 1). A total of 461 MDT discussions involving 455 patients were included in this study. The characteristics of patients and MDT are shown in Table 1. The median age was 59.0 [interquartile range (IQR) 50.0-65.0)] years, and 62.3\% were male. The other baseline characteristics were balanced among patients in the two groups, except more patients in the nonimplementation group without prior chemotherapy than in the implementation group. Most patients were recommended to receive comprehensive treatment (39.2\%), followed by local treatment (31.9\%), systemic treatment $(22 \cdot 8 \%)$, and only diagnosis $(6 \cdot 1 \%)$.

\section{MDT discussion}

The median length of time of MDT meeting conducted in involved medical centers was five years (IQR 37). Most MDT discussions were requested by surgeons ( $43 \cdot 4 \%)$, followed by medical oncologists ( $41 \cdot 6 \%)$, while a small proportion of patients were recommended by doctors from other departments. The aims of MDT discussion were mainly deciding further treatment plan (100\%); some cases also needed a definite diagnosis (22.6\%). Most MDT discussions (75.5\%) involved more than five disciplines. Most MDT discussions for every case (49.0\%) lasted more than 20 min followed by $10-20$ minutes (32.1\%), while some of them (18.9\%) lasted less than 10 minutes. The final MDT decisions were mostly made by medical oncologists $(62 \cdot 3 \%)$, followed by surgeons $(21 \cdot 5 \%)$, radiation or intervention therapists $(5 \cdot 0 \%)$, and others $(11 \cdot 3 \%)$.

\section{Implementation of MDT decision}

The final treatment of $395(85 \cdot 7 \%)$ patients was completely consistent with the MDT decision, and the final treatment of $66(14.3 \%)$ patients was inconsistent with the MDT decision. Therefore, according to the protocol, 395 and 66 patients were enrolled in the study and control groups, respectively. The other baseline characteristics of the two groups were basically balanced, besides the high proportion of chemotherapy-naïve patients in the nonimplementation group.

\section{Reasons for refusing to implement MDT decision}

Among the 66 patients who did not implement MDT decisions, 18 (27.3\%) had implemented MDT decisions in the initial stage, but could not continue the original MDT decision because of the change in disease condition later. However, only one of these patients agreed for the next MDT discussion when the condition changed. Further, $17(25.8 \%)$ patients or their families did not implement MDT decisions because they did not agree with the recommendation of MDT discussion. One patient could not afford the payment of MDT treatment and gave up. Other patients refused for other reasons, but the details were not recorded. 


\section{Association of implementation of MDT decisions with OS}

After a median follow-up of 15 months (IQR 10-21), 136 deaths occurred in all patients. The median OS was 23.0 months $(95 \% \mathrm{Cl} 20 \cdot 476-25 \cdot 524)$. The OS was $24 \cdot 0$ months $(95 \% \mathrm{Cl} 21 \cdot 872-26 \cdot 128)$ and $17 \cdot 0$ months (95\% Cl 12.167-21.833) in the MDT decision implementation group and nonimplementation group, respectively. The implementation of MDT decisions significantly reduced the risk of death in the univariate analysis ( $\mathrm{HR}=0.405,95 \% \mathrm{Cl} 0.274-0.599, P<0.001$; Table 2 and Figure. 2). Meanwhile, sex, tumor type, previous treatment of patients, and the way to pay medical care were also associated with OS in the univariate analysis (Table 2). The multivariable analysis tested whether the implementation of MDT decision was still significantly and independently associated with OS (HR $=0.425,95 \% \mathrm{Cl} 0.285-$ $0.633, P<0.001$, Table 2) when combined with the aforementioned factors. In addition, in the subgroup analysis, a significant difference was found in the survival analysis of patients with colorectal cancer, but no significant difference was found in patients with gastric cancer (Figure 3 and Figure 4).

\section{Discussion}

This study assessed whether the existing MDT discussion mode could bring survival benefits to patients with metastatic gastrointestinal cancer. Based on the requirements of ethics, it is difficult to design and carry out a randomized controlled study to answer this question directly. Therefore, the patients were categorized based on the implementation of the MDT discussion to evaluate the difference in OS, hoping to give an indirect answer. This was a major limitation of this study.

This study showed that the OS of patients was significantly longer in the implementation group than in the nonimplementation group, suggesting that the current MDT discussion played a positive role in patients with metastatic gastrointestinal cancer. This was the first prospective controlled study to try to answer this question. However, being a noninterventional study, selection bias might exist in patient selection. Although nearly 500 patients were included in the study, only 66 were in the nonimplementation group at the time of discontinuation of enrolment. The expected number of patients to be enrolled was not reached, and, therefore, the statistical comparison efficiency might decline. In addition, the study included as many factors as possible that might affect survival; however, still some features were not listed, such as specific issues discussed and specific location and number of tumours, thus increasing the probability of false-positive results.

In the subgroup analysis, the results showed that the survival benefit of patients with colorectal cancer who implemented MDT decisions was more obvious. Previous studies showed that in metastatic colorectal cancer, especially liver metastasis or lung metastasis, multidisciplinary treatment could significantly prolong survival[$[7,8]$, and this concept has been widely used in clinical practice. The results of the subgroup analysis of colorectal cancer in the present study showed that the analysis data were consistent with previous clinical findings $[9,10]$. More importantly, most patients with advanced colorectal cancer, whether they accepted MDT decisions, would still receive multidisciplinary comprehensive 
treatment in clinical practice. Therefore, the results of this study suggested that formal MDT discussion was still necessary for these patients.

In the subgroup analysis of gastric cancer, patients with MDT decision-making did not have significant survival benefits, which might be associated with the characteristics of metastatic gastric cancer. MDT of metastatic gastric cancer mainly focuses on retroperitoneal lymph node dissection, liver metastasis resection, hyperthermic intraperitoneal chemotherapy, and resection of Krukenberg's tumor[11]. However, whether the application of these treatments can prolong the survival of patients with metastatic gastric cancer is controversial[12, 13]. Although some treatment methods could not prolong the survival, systematic treatment combined with local treatment might improve the quality of life of patients with obstruction, perforation, bleeding, or pain. Unfortunately, the quality of life was not evaluated in this study, which was another limitation of this study.

The implementation rate of MDT decision in this study was similar to that reported in the past (80-90\%), indicating the trust of patients in MDT decision-making. The patient's background and disease characteristics were recorded, hoping to identify the main reasons associated with the refusal of MDT decision. This study showed that female patients had better compliance, which might be related to Chinese traditional culture. At the same time, patients who had not received previous treatment were more likely to refuse to implement MDT decisions than those who had received at least two-line treatment. This was a matter of caution because MDT decision-making might be more important for patients with newly diagnosed advanced cancer. Another important factor in not implementing MDT decision was the change in patient's condition in a later stage. Strictly speaking, this was not the patient's subjective intention to refuse to accept the original MDT decision. The present study found that almost all of the patients who failed to continue MDT decisions due to changes in their condition were not scheduled to receive another MDT discussion. This finding suggested that MDT discussion should be used not only in the initial treatment decision but also throughout the management of patient treatment. In addition, nearly half of the patients were not willing to talk about the reasons for denying the implementation of MDT decision. In general, patients were not invited to participate in MDT discussions. Hence, doctors might ignore patients' thoughts and concerns in MDT decision-making, affecting the acceptance and implementation of MDT decisions.

This study aimed to analyze what the best MDT mode was. The results showed that the participation of three to five disciplines and 15-20 mins of discussion time were suitable for completing MDT discussion in each case. Patients with colorectal cancer and patients who had not received previous treatment might benefit more from MDT discussion. An interesting phenomenon in the study was that most of the doctors who made the final MDT decision after discussion were oncologists. This might be related to the fact that all the participants involved in the discussion were patients with advanced cancer; oncologists need to focus more on the treatment process.

To sum up, despite limitations, the results of this study showed that the formal MDT discussions could significantly prolong the survival of patients with metastatic gastrointestinal cancer, especially for 
patients with metastatic colorectal cancer. At the same time, when the condition changes, it is necessary to schedule the next MDT discussion in time.

\section{Declarations}

\section{Ethical approval and informed consent}

The experimental protocol was approved by Beijing Cancer Hospital ethics committee, and was conducted in accordance with the Declaration of Helsinki and Good Clinical Practice. Informed consent was obtained from participants before enrolment.

\section{Consent for publication}

Not applicable

\section{Availability if data and materials}

The datasets generated during the current study are not publicly available due to limitation of ethical approval involving the patient data and anonymity but are available from the corresponding author upon reasonable request.

\section{Authors' contributions}

Jian Li and Lin Shen contributed to the study conception and design. All the authors provided resource of the data. Qi Zhang made the statistical analysis and wrote the manuscript. Jian Li and Lin Shen edited and reviewed the manuscript. All the authors have read and approved to published manuscript.

\section{Competing interests}

None of the authors declare any conflict of interest.

\section{Funding}

Not applicable.

\section{Acknowledgment}

We thank all the patients and their families for making this study possible.

\section{References}

1. Bray F, Ferlay J, Soerjomataram I, Siegel RL, Torre LA, Jemal A: Global cancer statistics 2018: GLOBOCAN estimates of incidence and mortality worldwide for 36 cancers in 185 countries. $C A$ Cancer J Clin 2018, 68(6):394-424. 
2. Dec: A Policy Framework for Commissioning Cancer Services : A Report by the Expert Advisory Group on Cancer to the Chief Medical Officers of England and Wales.

3. Øvretveit, John: Five ways to describe a multidisciplinary team. Hol Medicine 1996, 10(2):163-171.

4. El Saghir NS, Keating NL, Carlson RW, Khoury KE, Fallowfield L: Tumor Boards: Optimizing the Structure and Improving Efficiency of Multidisciplinary Management of Patients with Cancer Worldwide. American Society of Clinical Oncology Educational Book 2014(34):e461-e466.

5. Fennell ML, Das IP, Clauser S, Petrelli N, Salner A: The organization of multidisciplinary care teams: modeling internal and external influences on cancer care quality. Journal of the National Cancer Institute Monographs 2010, 2010(40):72-80.

6. Ottevanger N, Hilbink M, Weenk M, Janssen R, Vrijmoeth T, de Vries A, Hermens R: Oncologic multidisciplinary team meetings: evaluation of quality criteria. Journal of evaluation in clinical practice 2013, 19(6):1035-1043.

7. Chen CH, Hsieh MC, Lao WT, Lin EK, Lu YJ, Wu SY: Multidisciplinary team intervention associated with improved survival for patients with colorectal adenocarcinoma with liver or lung metastasis. American journal of cancer research 2018, 8(9):1887-1898.

8. Keller DS, Berho M, Perez RO, Wexner SD, Chand M: The multidisciplinary management of rectal cancer. Nat Rev Gastroenterol Hepatol 2020, 17(7):414-429.

9. Munro A, Brown M, Niblock P, Steele R, Carey F: Do Multidisciplinary Team (MDT) processes influence survival in patients with colorectal cancer? A population-based experience. $B M C$ cancer 2015, 15:686.

10. Hsu Y-H, Kung P-T, Wang S-T, Fang C-Y, Tsai W-C: Improved patient survivals with colorectal cancer under multidisciplinary team care: A nationwide cohort study of 25,766 patients in Taiwan. Health Policy 2016, 120(6):674-681.

11. Beom SH, Choi YY, Baek SE, Li SX, Lim JS, Son T, Kim HI, Cheong JH, Hyung WJ, Choi SH et al: Multidisciplinary treatment for patients with stage IV gastric cancer: the role of conversion surgery following chemotherapy. BMC Cancer 2018, 18(1):1116.

12. Fujitani K, Yang HK, Mizusawa J, Kim YW, Terashima M, Han SU, Iwasaki Y, Hyung WJ, Takagane A, Park DJ et al: Gastrectomy plus chemotherapy versus chemotherapy alone for advanced gastric cancer with a single non-curable factor (REGATTA): a phase 3 , randomised controlled trial. The Lancet Oncology 2016, 17(3):309-318.

13. Montagnani F, Crivelli F, Aprile G, Vivaldi C, Pecora I, De Vivo R, Clerico MA, Fornaro L: Long-term survival after liver metastasectomy in gastric cancer: Systematic review and meta-analysis of prognostic factors. Cancer treatment reviews 2018, 69:11-20.

\section{Tables}


Table 1

Characteristics of patients and multidisciplinary team

\begin{tabular}{ll} 
Implementation group & Nonimplementation group \\
\hline$(N=395), N(\%)$ & $(N=66), N(\%)$
\end{tabular}

Age, year

$\begin{array}{llll}<50 & 97(24 \cdot 6) & 15(22 \cdot 7) & 0 \cdot 928 \\ 50-69 & 249(63 \cdot 0) & 42(63 \cdot 6) & \\ \geq 70 & 49(12 \cdot 4) & 9(13 \cdot 6) & 0.063 \\ \text { Sex } & & & \\ \text { Male } & 240(60 \cdot 8) & 18((27 \cdot 3) & 0.505 \\ \text { Female } & 155(39 \cdot 2) & & \\ \text { Tumor type } & & 24(36 \cdot 4) & \\ \text { Gastric cancer } & 118(29 \cdot 9) & 34(51 \cdot 5) & 8(12 \cdot 1)\end{array}$

Number of prior chemotherapy lines

\begin{tabular}{llll}
\hline 0 & $150(38 \cdot 0)$ & $33(50 \cdot 0)$ & $0 \cdot 030$ \\
\hline $156(39 \cdot 5)$ & $27(40 \cdot 9)$ & & \\
$89(22 \cdot 5)$ & $6(9 \cdot 1)$ & & $0 \cdot 972$ \\
\hline Aim of MDT discussion & & $15(22 \cdot 7)$ & \\
\hline Diagnose and treatment & $89(22 \cdot 5)$ & $51(77 \cdot 3)$ & $0 \cdot 714$ \\
\hline Treatment & $306(77 \cdot 5)$ & & \\
\hline MDT decision & & $3(4 \cdot 5)$ & \\
\hline Diagnose & $25(6 \cdot 3)$ & $19(28 \cdot 8)$ & \\
\hline Local treatment & $128(32 \cdot 4)$ &
\end{tabular}

Abbreviations:

MDT, multidisciplinary team; mins, minutes;

*Other tumor types included esophageal squamous carcinoma $(n=4)$, appendiceal cancer $(n=1)$, pancreatic cancer $(n=6)$, cholangiocarcinoma $(n=8)$, gastrointestinal stroma tumor $(n=2)$, and neuroendocrine tumor $(n=31)$. 


\begin{tabular}{|c|c|c|c|}
\hline & Implementation group & Nonimplementation group & $P$ \\
\hline & $(N=395), N(\%)$ & $(N=66), N(\%)$ & \\
\hline Systemic treatment & $91(23 \cdot 0)$ & $14(21 \cdot 2)$ & \\
\hline Comprehensive treatment & $151(38 \cdot 3)$ & $30(45 \cdot 5)$ & \\
\hline Timing of MDT & & & \\
\hline First-time consultation & $138(34 \cdot 9)$ & $23(34 \cdot 8)$ & 0.989 \\
\hline Further consultation & $257(65 \cdot 1)$ & $43(65 \cdot 2)$ & \\
\hline Way to pay medical care & & & \\
\hline Medical insurance & $314(79 \cdot 5)$ & $51(77 \cdot 3)$ & 0.796 \\
\hline Self-payment & $72(18 \cdot 2)$ & $14(21 \cdot 2)$ & \\
\hline Missing data & $9(2 \cdot 3)$ & $1(1 \cdot 5)$ & \\
\hline Number of involved discipl & & & \\
\hline $3-5$ & $92(23 \cdot 3)$ & $21(31 \cdot 8)$ & 0.136 \\
\hline$>5$ & $303(76 \cdot 7)$ & $45(68 \cdot 2)$ & \\
\hline Duration of MDT & & & \\
\hline$\leq 10$ mins & $75(19 \cdot 0)$ & $12(18 \cdot 2)$ & 0.542 \\
\hline $10-20$ mins & $123(31 \cdot 1)$ & $25(37 \cdot 9)$ & \\
\hline$>20$ mins & $197(49 \cdot 9)$ & $29(43.9)$ & \\
\hline Abbreviations: & & & \\
\hline MDT, multidisciplinary tean & ns, minutes; & & \\
\hline $\begin{array}{l}{ }^{*} \text { Other tumor types includec } \\
\text { pancreatic cancer }(n=6) \text {, c } \\
\text { neuroendocrine tumor }(n=\end{array}$ & $\begin{array}{l}\text { phageal squamous carc } \\
\text { ngiocarcinoma }(n=8), \mathrm{g}\end{array}$ & $\begin{array}{l}\text { a }(n=4) \text {, appendiceal cance } \\
\text { intestinal stroma tumor }(n=\end{array}$ & $\begin{array}{l}\text { 1), } \\
\text { nd }\end{array}$ \\
\hline
\end{tabular}


Table 2

Univariate and multivariate analyses of overall survival

Univariate analysis

$\mathrm{HR} \quad 95 \% \mathrm{Cl}$

Factors

Age, year

$\geq 70$

$50-69$

$<50$

1

$0.675 \quad 0.468-\quad 0.036$

0.975
1

$\begin{array}{lll}0.909 & \begin{array}{l}0.497- \\ 1.662\end{array} & 0.757\end{array}$

$1.014 \quad 0.604-\quad 0.958$

$1 \cdot 703$
Sex

Male

Female
Multivariate analysis

$\mathrm{HR} \quad 95 \% \mathrm{Cl} \quad P$

1

Tumor site

1

$0 \cdot 291$

$0.203-<0.001$

0.417

Gastric cancer

Colorectal cancer

$\begin{array}{ll}0.331 & 0.159 \\ & 0.691\end{array}$

$0.159-<0.001$

$\begin{array}{ll}0.331 & 0.159 \\ & 0.691\end{array}$

1

$0 \cdot 400$

$0 \cdot 256-$

$<0.001$

Other cancer

$0 \cdot 370$

$0 \cdot 170-$

$0 \cdot 801$

Number of prior chemotherapy

lines

$\begin{array}{llll}\geq 2 & 1 & & \\ 1 & 1.541 & 0.923- & 0.098 \\ & & 2.572 & \\ 0 & 1.014 & 0.599- & 0.958 \\ & & 1.717 & \end{array}$

Aim of MDT discussion

Diagnose and treatment

1

Treatment

$\begin{array}{lll}0.990 & \begin{array}{l}0.659- \\ 1.489\end{array} & 0.963\end{array}$

Abbreviations: MDT, multidisciplinary team; mins, minutes;

*Other tumor types included esophageal squamous carcinoma $(n=4)$, appendiceal cancer $(n=1)$, pancreatic cancer $(n=6)$, cholangiocarcinoma $(n=8)$, gastrointestinal stroma tumor $(n=2)$, and neuroendocrine tumor $(n=31)$. 


\section{Univariate analysis}

\section{Multivariate analysis}

MDT decision

Diagnose

1

local treatment

$1 \cdot 033$

$0 \cdot 490-$

0.932

$2 \cdot 178$

Systemic treatment

$0 \cdot 651$

0.292-

$0 \cdot 294$

$1 \cdot 453$

Comprehensive treatment

$\left.\begin{array}{lll}0.632 & 0.299- & 0.230 \\ & 1.337\end{array}\right]$

Previous treatment of patients

Chemotherapy previous 1

Chemotherapy naive

$0 \cdot 445$

$0.302-\quad<0.001$ 0.655

1

0.504

$0 \cdot 334-$

0.761

$0 \cdot 001$

Way to pay medical care

Self-payment

1

1

Medical insurance

$0 \cdot 272$

$\begin{array}{ll}0.183- & <0.001 \\ 0.406\end{array}$

0.633

$0 \cdot 384-$

$1 \cdot 044$

0.073

Missing data

Number of involved disciplines

$\begin{array}{llll}3-5 & 1 & & \\ >5 & 1.041 & 0.730- & 0.823 \\ & & 1.485 & \end{array}$

Implemented decision

Non-implemented 1

1

Implemented

$0 \cdot 405$

$0.274-\quad<0.001$

0.599

$0 \cdot 425$

$0 \cdot 285-$

0.633

Duration of MDT

$\leq 10$ mins

1

10-20 mins

$1 \cdot 509$

$0 \cdot 944-$

$2 \cdot 410$

0.085

Abbreviations: MDT, multidisciplinary team; mins, minutes;

*Other tumor types included esophageal squamous carcinoma $(n=4)$, appendiceal cancer $(n=1)$, pancreatic cancer $(n=6)$, cholangiocarcinoma $(n=8)$, gastrointestinal stroma tumor $(n=2)$, and neuroendocrine tumor $(n=31)$. 


\begin{tabular}{|c|c|c|c|}
\hline & \multicolumn{2}{|c|}{ Univariate analysis } & \multirow[t]{2}{*}{ Multivariate analysis } \\
\hline$>20$ mins & 0.916 & $\begin{array}{l}0.567- \\
1.478\end{array}$ & \\
\hline \multicolumn{4}{|c|}{ Abbreviations: MDT, multidisciplinary team; mins, minutes; } \\
\hline \multicolumn{4}{|c|}{$\begin{array}{l}\text { *Other tumor types included esophageal squamous carcinoma }(n=4) \text {, appendiceal cancer }(n=1) \text {, } \\
\text { pancreatic cancer }(n=6) \text {, cholangiocarcinoma }(n=8) \text {, gastrointestinal stroma tumor }(n=2) \text {, and } \\
\text { neuroendocrine tumor }(n=31) \text {. }\end{array}$} \\
\hline
\end{tabular}

\section{Figures}

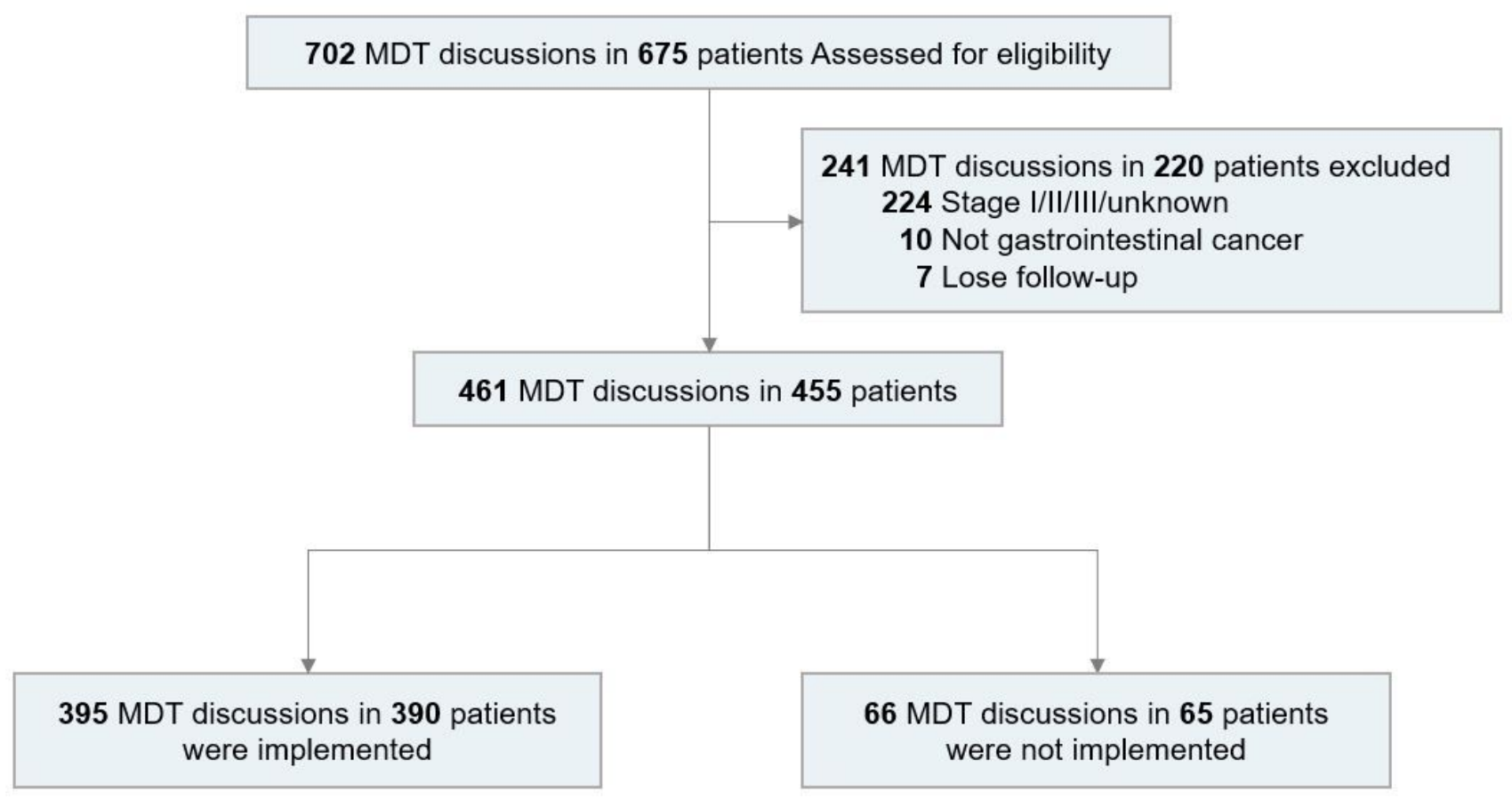

Figure 1

Study Flowchart

MDT indicates multidisciplinary team. 
No. at risk

Implementation group 395

Nonimplementation group 66

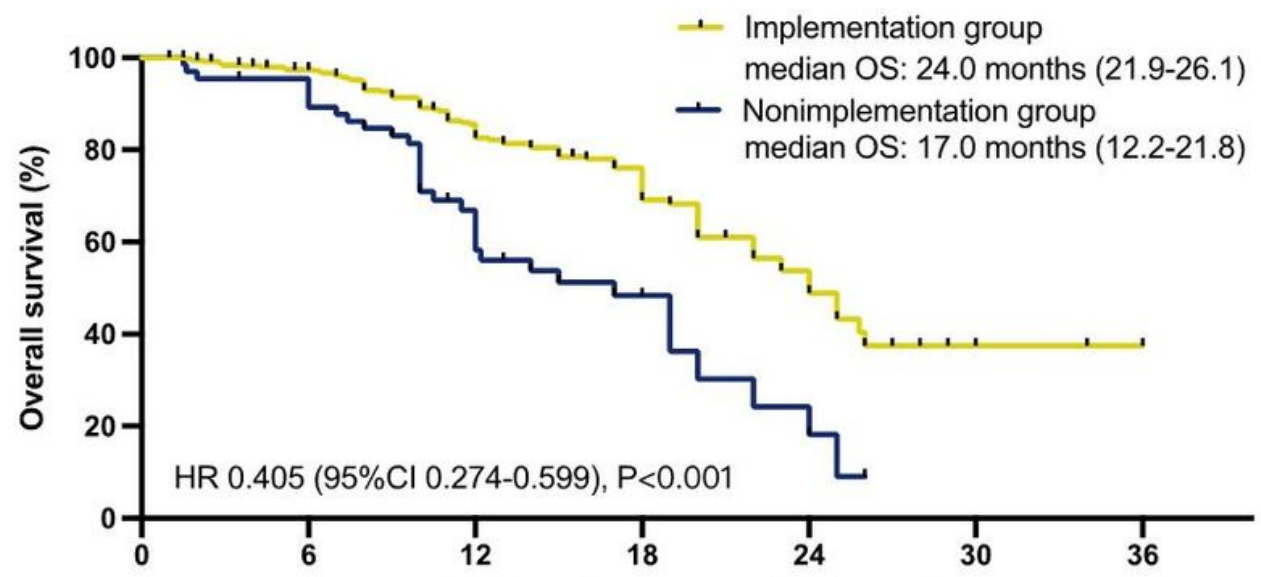

Time since MDT discussion (months)

$\begin{array}{cccccc}372 & 227 & 110 & 33 & 4 & 1 \\ 62 & 31 & 12 & 4 & 0 & 0\end{array}$

\section{Figure 2}

Kaplan-Meier curves of overall survival depending on the implementation of MDT decisions in the whole population.

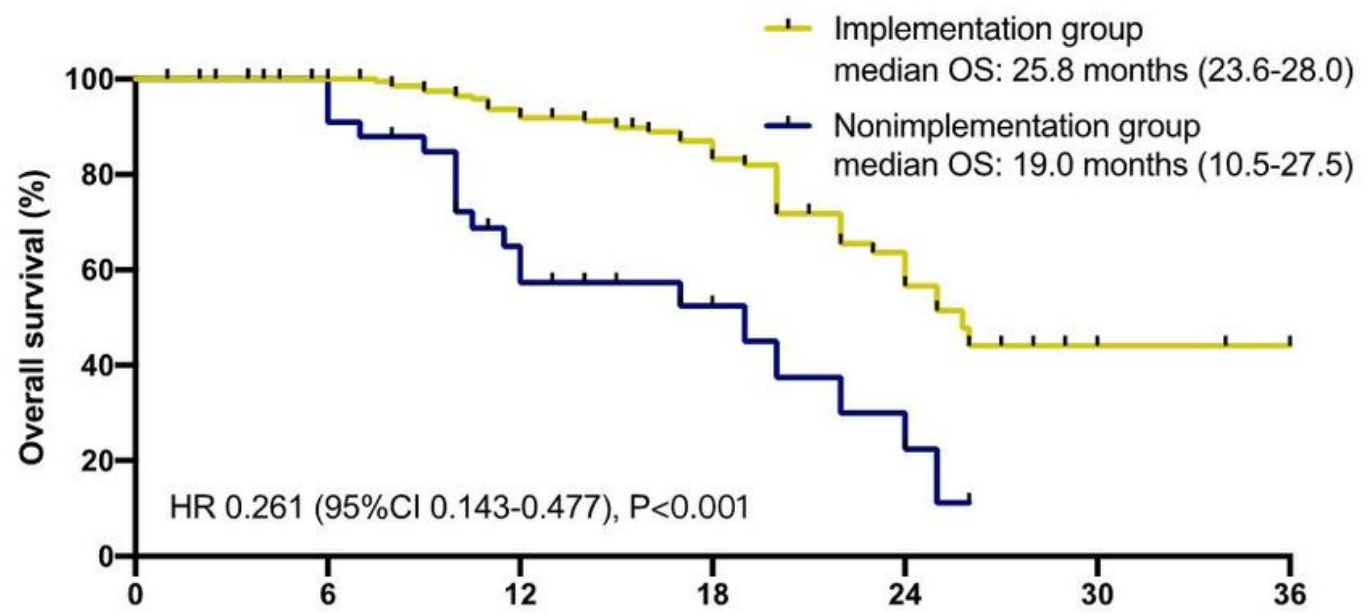

No. at risk

Implemented group

Nonimplemented group
233

34

Time since MDT discussion (months)

223

33

\begin{abstract}
160
\end{abstract}
17
90

9

\begin{abstract}
27
\end{abstract}
4
4

0
1

0 


\section{Figure 3}

Kaplan-Meier curves of overall survival depending on the implementation of MDT decisions in the colorectal cancer subgroup.

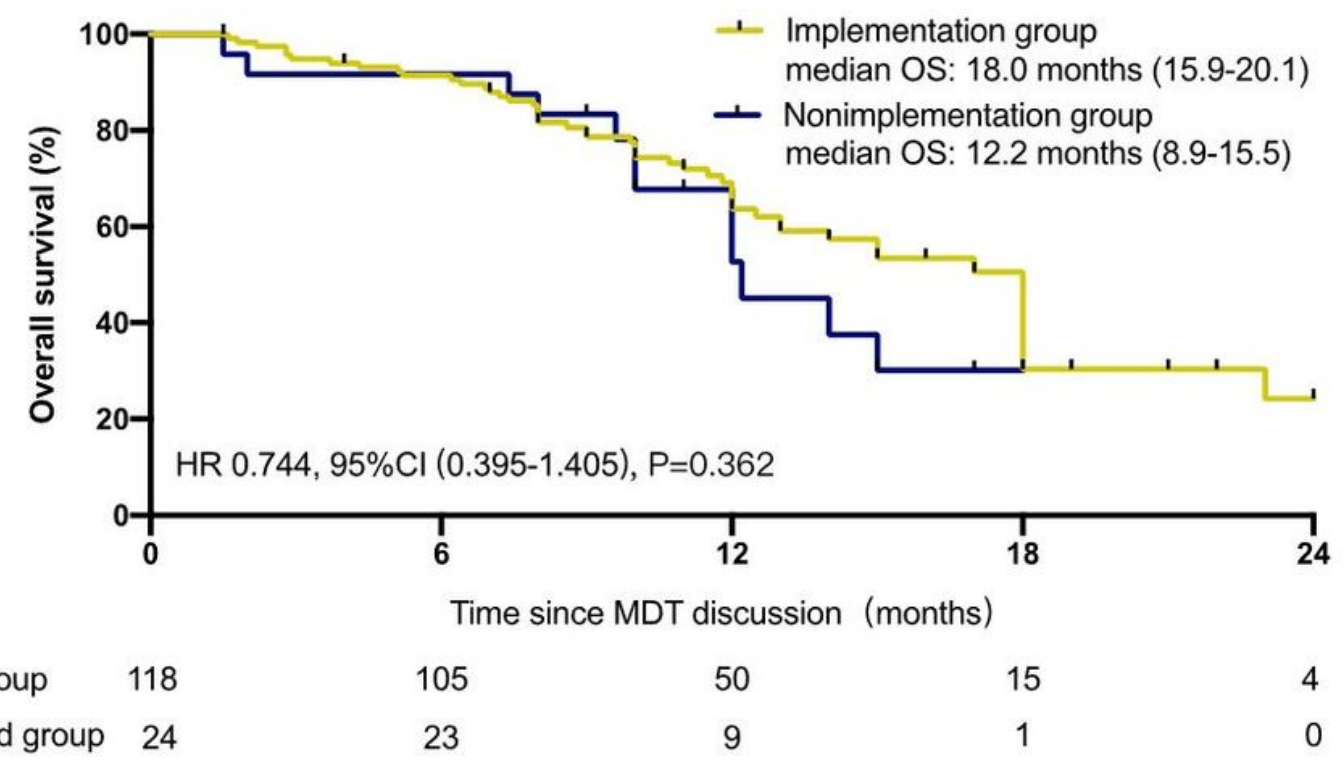

\section{Figure 4}

Kaplan-Meier curves of overall survival depending on the implementation of MDT decisions in the gastric cancer subgroup.

\section{Supplementary Files}

This is a list of supplementary files associated with this preprint. Click to download.

- Protocol.docx 